\title{
CONTRIBUTION OF MASSIVE NEUTRINO ON LARGE SCALE STRUCTURES
}

\author{
P.R. Dhungel*, S.K. Sharma*** and U. Khanal*k** \\ *Little Angles College, Hattiban, Lalitpur, Nepal. \\ **BPKMPOSM Development Board, Ministry of Environment, Science and Technology, Singhadurbar, Kathmandu, Nepal. \\ *****entral Department of Physics, Tribhuvan University, Kirtipur, Kathmandu, Nepal.
}

\begin{abstract}
Sizes and masses of the Neutrino Structures calculated with different combination of parameters involved has shown interesting variation with remarkable shifting of position of peak value. The peak position of $\left(\xi R_{H 0} / R_{e s c}\right)$ shifts from $\xi=x / x_{0}=0.847$ to 1.632 , where $x=m / T$. Accordingly, the peak of the mass of neutrino structures also shifts.
\end{abstract}

Key words: Neutrino; Large scale stru ctures; Free str eaming; Jeans mass; Momentum distribution.

\section{INTRODUCTION}

The enigmatic neutrino has been generating great deal of interest in cosmology for a long time. Regarding the number of neutrino species e.g., calculations of primordial nuclear abundances in cosmology had arrived at the number of neutrino species $\mathrm{N}_{4}<4\left[^{1}\right]$ long before it was experimentally established at CERN $\left[{ }^{2}\right]$; the current value is $\mathrm{N}_{\mathrm{y}}=2.994 \pm 0.012$ $\left[{ }^{3}\right]$. Similar calculations set the baryon to photon ratio at $\sim$ $4.7 \times 10^{-10}$, which in turn has determined the present baryon density in the Universe to be $\Omega_{B} h^{2}=0.0223$, where $\Omega_{B}=\rho_{B} /$ $\rho_{c}$ is the present baryon density in units of the critical density, and $h$ is the present value of the Hubble parameter in units of $100 \mathrm{~km} / \mathrm{s} / \mathrm{Mpc}$ that is expected to be $\mathrm{h} \sim 0.73\left[{ }^{3}\right]$. Writing the present neutrino density as $\Omega \mathrm{h}^{2}=\mathrm{m}_{\mathrm{eV}} / 31$, where $\mathrm{m}_{\mathrm{eV}}$ is the average of the three rest-masses of the neutrino in $\mathrm{eV}$, it is easily seen that the $31 \mathrm{eV}$ neutrino will close the Universe; this closure mass is referred to as the Cowsik- McClelland bound $\left[{ }^{4}\right]$, although in their paper they had used a smaller value of $\Omega$ and two neutrino species to arrive at $8 \mathrm{eV}$. Termain and Gunn $\left[{ }^{5}\right]$ set the limit that neutrino of $\mathrm{m}_{\mathrm{v}}<1 \mathrm{MeV}$ cannot reside in the halo of galaxy to contribute significantly to the dark matter. Bond et al $\left[^{6}\right]$ calculated the maximum Jeans mass for structures of neutrino to be $\mathrm{M}_{\mathrm{v}} \mathrm{m} \approx 1.2 \times 10^{17} \mathrm{~m}_{\mathrm{eV}}^{-2} \mathrm{M}_{\mathrm{t}}$, where $\mathrm{m}_{\mathrm{ev}}$ is the neutrino mass in $\mathrm{eV}$.

As recent results show that the mass difference between neutrino species is very slight, $\Delta \mathrm{m}^{2} \approx 10^{-5} \mathrm{eV}^{2}$, in this paper, we consider three neutrino species of similar mass. Comparing the baryon and neutrino densities, we see that even a $0.7 \mathrm{eV}$ neutrino will have dominated over baryon by now. Rather than whether the neutrino is massive, the question at present is how massive it is; again cosmology provides the most stringent limit $\Sigma \mathrm{m}_{\mathrm{v}}<0.7 \mathrm{eV}\left[{ }^{7}\right]$. So the neutrino appears as a very important component of the Universe. Consequently, it should have a strong bearing on structure formation. It decouples at very early times, and then evolves as a totally independent component that interacts only gravitationally. Indeed the filamentary structures, sheets, walls and voids as exposed by various surveys point towards dissipationless collapse of clouds of particles like neutrinos into Zeldovich pancakes $\left[{ }^{8}\right]$ at some stage of the evolution. Although the cosmological neutrino has been studied extensively, we feel that our analysis sheds some further insight on structure formation.

The momentum of a freely moving particle in the FriedmannRobertson-Walker spacetime is redshifted by the expansion, i.e., the comoving momentum $\mathrm{pa}=\mathrm{y}$ remains constant, where $\mathrm{p}$ is the momentum and a the scale factor. Using $\mathrm{v}=\mathrm{p} / \mathrm{E}$ for the velocity and the Einstein energy-momentum relation, $\mathrm{a}^{2} \mathrm{E}^{2}$ $=\mathrm{p}^{2} \mathrm{a}^{2}+\mathrm{m}^{2} \mathrm{a}^{2}=\mathrm{y}^{2}+\mathrm{x}^{2}$, we see that Eva remains constant during expansion, and as the number density scales as $\mathrm{a}^{-3}, \rho v a^{4}$ also remains constant where $\rho$ is the density. As the light neutrinos decouple at the very high temperature of $\mathrm{T} \approx 1 \mathrm{MeV}$ while still extremely relativistic (ER), they are essentially in free fall since then. So their number density is always distributed as

$$
d n=\frac{g}{2 \pi^{2}}\left(\frac{m}{x}\right)^{3} \frac{y^{2}}{e^{y}+1} d y
$$

where $\mathrm{g}$ is the number of spin degeneracy (six, for the three $v-\bar{v}$ pairs), and we have used the fact that $\mathrm{T} \sim 1 / \mathrm{a}$; Planck units in which $\mathrm{G}=\mathrm{c}=\mathrm{k}_{\mathrm{B}}=\hbar=1$ are used throughout this work. $A S E \sim p ~ \gg m$ in the extreme relativistic (ER) regime, Eq. (1) represents the Fermi-Dirac distribution. In the nonrelativistic (NR) case however, $E \sim p^{2} / 2 m+m$, and $E q$. (1) is no more a Fermi-Dirac distribution.

Anthor for Comespondence: Prof. Dr. Udayaraj Khanal, Central Department of Physics, Tribhuvan University, Kirtipur, Kathmandı, Nepal. 


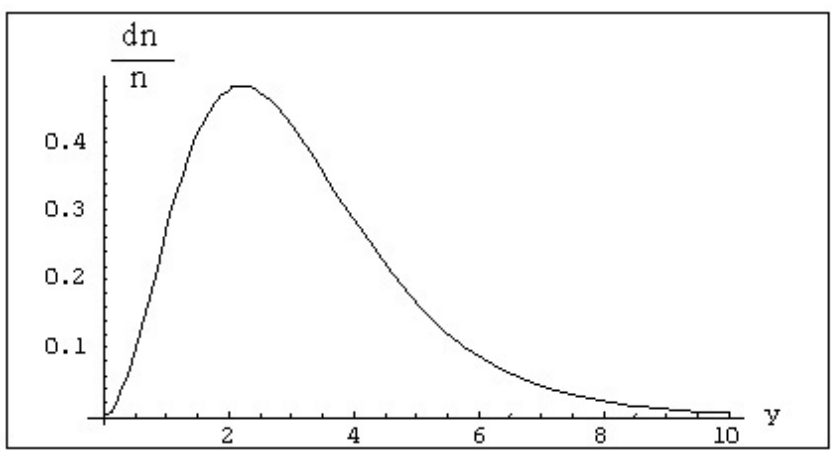

Fig.-1: Momentum distribution of the free cosmic neutrino as a function of $\mathrm{y}=\mathrm{pa}=\mathrm{p} / \mathrm{T}_{\mathrm{y}}$. The distribution is maximum at $\mathrm{y}_{\max }=$ 3.131 , and other characteristic values are $\mathrm{y}_{\operatorname{ms}}=\left\langle\mathrm{y}^{2}\right\rangle, 1 / 2=3.597$ and $\mathrm{y}_{\text {rims }}=\left\langle 1 / \mathrm{y}^{2}\right\rangle-1 / 2=1.631$. Such momentum distribution give rise to a spectrum of neutrino structure scales

Integrating over $\mathrm{y}$ from 0 to $\infty$ gives the number density $n=\frac{g}{2 \pi^{2}} \Gamma(3) \eta(3)\left(\frac{m}{x}\right)^{3}$ where $\Gamma$ and $\eta$ are the gamma and the Riemann eta functions respectively [ $\left.{ }^{9}\right]$; also, $\eta(n)=\left(1-2^{1-n}\right) \zeta(n)$. Thus we can write down the expectation value of any regular function as $\langle f(y)\rangle=\frac{1}{\Gamma(3) \eta(3)} \int_{0}^{\infty} d y \frac{y^{2}}{e^{y}+1} f(y)$. The width of the momentum distribution shown in Fig. -1 is characterized by $\mathrm{y}_{\max }$ $=3.131$ where the distribution is maximum Other characteristic

values like the mean $\mathrm{y}_{\operatorname{mex}}=\langle\mathrm{y}\rangle=\frac{\Gamma(4) \eta(4)}{\Gamma(3) \eta(3)}=\frac{7 \zeta(4)}{2 \zeta(3)}=3.151$, the root- mean-square $\mathrm{y}_{\mathrm{ms}}=\left\langle\mathrm{y}^{2}\right\rangle^{1 / 2}=\sqrt{\frac{15 \zeta(5)}{\zeta(3)}}=3.597$, the harmonic mean $\mathrm{y}_{\mathrm{km}}=\left\langle\mathrm{y}^{1}\right\rangle^{-1}=\frac{3 \zeta(3)}{\zeta(2)}=2.192$, and the root harmonic mean square $\mathrm{y}_{\text {trms }}=\left\langle\mathrm{y}^{2}\right\rangle^{-1 / 2}=\sqrt{\frac{\Gamma(3) \eta(3)}{\Gamma(1) \eta(1)}}=\sqrt{\frac{3 \zeta(3)}{2 \ln (2)}}$ $=1.613$ are alsouseful to describe the width of the distribution. The particle speed is $\mathrm{v}=\mathrm{p} / \mathrm{E}=\mathrm{y} / \sqrt{y^{2}+x^{2}}$. In the ER case as $\mathrm{y}>\mathrm{x}$, v(y) $\rightarrow \mathrm{x}^{2} / 2 \mathrm{y}^{2}+\ldots$. , giving v vem $\rightarrow 1-\frac{1}{2}\left(\frac{x}{y_{\text {rhms }}}\right)^{2}$ $\rightarrow \mathrm{v}_{\mathrm{rtms}}$; also, $\mathrm{v}_{\mathrm{rm}}=\langle 1 / \mathrm{v}\rangle^{-1} \rightarrow \mathrm{v}_{\mathrm{rmms}}$. Thus, we see that $\mathrm{v}_{\mathrm{rtm}}$ is the more representative speed in the ER regime. These ideas can be used for a bosonic system as well by replacing $\eta$ with $\zeta$. But $\zeta(1)=\infty$, so $\mathrm{y}_{\mathrm{rtms}}$ (boson) $=0$, indicative of the liability of bosons condensing into a zero momentum state. Although a zero momentum state is strictly forbidden for a fermionic system, as $\eta(1)=\ln (2)$, a low momentum condensate characterized by the harmonic mean values is possible. The rms energy is given by $a^{2} \mathrm{E}_{m s}^{2}=y_{m s}^{2}+x^{2}$, and another characteristic value of the energy is the velocity averaged momentum $\left.a^{2} E_{v}^{2}=\left\langle y^{2}\right\rangle / 1 / v^{2}\right\rangle=y_{r w s}^{2}\left(1+x^{2} / \gamma_{r k m s}^{2}\right)$ Obviously, as a $\rightarrow 0, \mathrm{E} \rightarrow \infty$

Any macroscopic quantity that depends on the momentum distribution will be sensitive to the averaging process. This will be even more so in the relativistic regime. In this paper, we look into such effects and investigate the possible distribution of the sizes of neutrino structures. In particular, we would like to determine whether smaller neutrino structures could have formed in the very early Universe. In the next Section, we apply these ideas to the gravitating neutrino spheres, and compare the distributions of Keplerian, Virial and Jeans and the free streaming scales.

\section{NEUTRINO STRUCTURE SCALES}

To investigate the scales of neutrino structures, let us rewrite the Friedmann equation in terms of the Hubble radius $\mathrm{R}_{\mathrm{H}}=$ $1 / \mathrm{H}$ in the following form,

$$
\frac{R_{H_{0}}^{2}}{R_{H}^{2}}=\frac{H^{2}}{H_{0}^{2}}=\frac{R_{H_{0}}^{2}}{\xi^{2}}\left(\frac{d \xi}{d t}\right)^{2}=\frac{\rho}{\rho_{c 0}}+\frac{1-\Omega}{\xi^{2}}=\frac{f(\xi)}{\xi^{4}}
$$

here, the subscript 0 represents the respective values at some reference time $t_{0}$ and $\rho_{c o}$ is the critical density; $p$ is the total energy density contributed by matter and radiation, while $(1-\Omega)$ may be considered to be the energy density due to curvature; in particular, $\mathrm{f}(\xi)=\Omega_{\Delta 0} \xi^{4}+\left(1-\Omega_{0}\right) \xi^{2}+\Omega_{\mathrm{d} 0} \xi+\Omega_{\gamma 0}+$ $\left.\Omega_{\text {vrest } 0}\left(\sqrt{\left(\xi^{2}+\left(y / x_{0}\right)^{2}\right.}\right)\right)$, where $\xi=\mathrm{x} / \mathrm{x}_{0}=\mathrm{a} / \mathrm{a}_{0}=\mathrm{T}_{0} / \mathrm{T},\left(\Omega_{\Lambda, \mathrm{d}, \mathrm{\gamma}, \mathrm{v}}\right)$ are the respective contributions to the total density (in units of critical density) by the cosmological constant, pressureless dust, photon, neutrino, etc. In the way we have written the neutrino density, we need not make the distinction between radiation and matter domination as its density scales appropriately in the respective regimes, i.e.,

$\rho_{u} / \rho_{c 0} \rightarrow \begin{cases}\Omega_{u 0} / \xi^{4}, & \xi \rightarrow 0, \\ \Omega_{u \text { rest } 0} / \xi^{3}, & \xi \rightarrow 0,\end{cases}$

The ratio of neutrino and photon densities is,

$$
\frac{\Omega_{v}}{\Omega_{y}}=\frac{\rho_{v}}{\beta_{7}}=\left(\frac{T_{v}}{T_{7}}\right)^{+} \frac{3 \zeta(3)}{4 \zeta(4)} x\left\langle\sqrt{1+(y / x)^{2}}\right\rangle=\frac{\Omega_{v r a b}}{\Omega_{7}}\left\langle\sqrt{1+(y / x)^{2}}\right\rangle
$$

the temperature ratio $\mathrm{T}_{v} / \mathrm{T}_{\gamma}$ is unity at very high temperature and is $(4 / 11)^{1 / 3}$ at temperatures below the neutrino decoupling and $e^{+}-e$ annihilation, i. e. $T v<1 \mathrm{MeV}$; the density ratio rises linearly in the NR region. The temperature of photonneutrino equality can be determined by setting Eq.(4) equal to unity, whence one finds $x_{\mathrm{eq}}=3.179$. No matter what the mass, neutrino density will start to dominate over radiation when the thermal energy goes below one-third of the rest mass energy. As we will be using different means of $y$, and $x_{\mathrm{eq}}$ will not be the same for all means, a more versatile expression at equality is 


$$
\frac{y / x_{e q}}{\sqrt{1+\left(y / x_{e q}\right)^{2}}}=\left(\frac{T_{u}}{T_{y}}\right)^{4} \frac{3 \zeta(3)}{4 \zeta(4)} y
$$

The most important length scale in cosmological context is the Hubble radius; the ratio of the comoving Hubble radii at equality and at arbitrary time is given by

$$
\left(\frac{R_{H} / a_{0}}{R_{H} / a}\right)^{2}=\left(\frac{\xi R_{H}}{R_{H}}\right)^{2}=\frac{f(\xi)}{\xi^{2}}
$$

The case $\Lambda=0$ : the limit of this ratio as $\xi \rightarrow \infty$ is $\left(1-\Omega_{0}\right)$, which means that in the open universe with $\Omega_{0}<1$, the maximum comoving Hubble radius can be $\left(\frac{\boldsymbol{E}_{n}}{a}\right) \rightarrow \frac{\boldsymbol{R}_{n} / \alpha_{0}}{\sqrt{1-\Omega_{0}}}$; in a flat universe with $\Omega_{0}=1,\left(\mathrm{R}_{\mathrm{H}} / \mathrm{a}\right)_{\max } \rightarrow \infty$; for a closed universe, the maximum Hubble radius is itself finite. If $\Lambda>0$, then $\left\langle\mathrm{R}_{\mathrm{H}} / \mathrm{a}\right)_{\max } \rightarrow \infty$. A length scale relevant to a gravitationally bound gaseous system is the radius where the escape speed becomes equal to the average random speed of the gas particles viz., $\mathrm{v}^{2}=2 \mathrm{M} / \mathrm{R}_{\mathrm{exc}}=\frac{8 \pi}{3} a R_{\mathrm{w}}$, or $\frac{1}{R^{2}}=\frac{8 \pi g}{3 v^{\prime}}$. As the smooth background of photons will not contribute to the potential well, here $p=p_{v}$. We can use the expression for critical density to write the ratio of the comoving Hubble and escape radii in a suggestive form that looks similar to Eq. (6):

$$
\left(\frac{\xi R_{B_{0}}}{R_{e x}}\right)^{2}=\frac{f_{c u}(\xi)}{\xi^{2}}
$$

where

$$
f_{e s c}(\xi)=\Omega_{u \text { rest } 0}\left({\left.\sqrt{\xi^{2}+\left(y / x_{0}\right.}\right)^{2}}^{2} / v^{2}\right.
$$

We can also define the Keplerian radius $\mathrm{R}_{\mathrm{K}}$ by $\frac{1}{R_{\kappa}^{2}}=\frac{4 \pi \rho}{3 v_{r \alpha}^{2}}=\frac{1}{2 R^{2}}$ with the understanding that $\mathrm{v}$ stands for the rotational speed; obviously, any particle orbiting with $v_{\mathrm{rot}}+\mathrm{v}_{\mathrm{ms}}>\mathrm{v}_{\mathrm{esc}}$ will escape. Similarly, the virial radius given by $2\left\langle\mathrm{v}^{2}\right\rangle=$ $\mathrm{M}<1 / \mathrm{R}_{\mathrm{v}}>$ leads to $\frac{1}{R^{2}}=\frac{4 \pi \rho}{6 v^{2}}=\frac{1}{4 R^{2}}$. Another such scale, the Jeans radius, is given by $\frac{1}{R_{j}^{2}}=\frac{4 \rho}{\pi v^{2}}=\frac{3}{2 \pi^{2} R_{e x}^{2}}$. All these length scales are some numerical multiples of $R_{\text {esc }}$

In any spectral analysis, it is the wavenumber $\mathrm{k} \sim 1 / \mathrm{R}$ that is fundamental, and similarly, in the Jeans analysis, the Jeans wavenumber $\mathrm{k}_{\mathrm{J}}$ should be averaged over all momenta. We have seen that Resc is the underlying quantity, so let us look into the behaviour of $1 / R^{2}{ }_{\text {esc }}$. There are a number of ways of averaging Eq. (7):

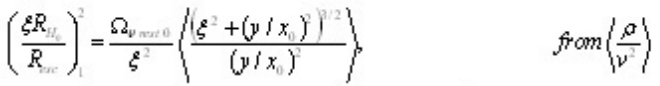

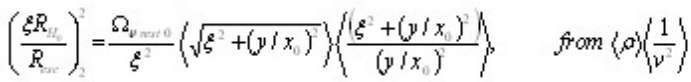

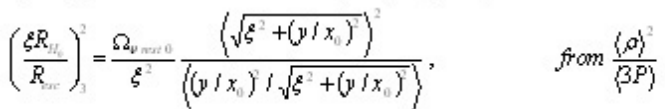

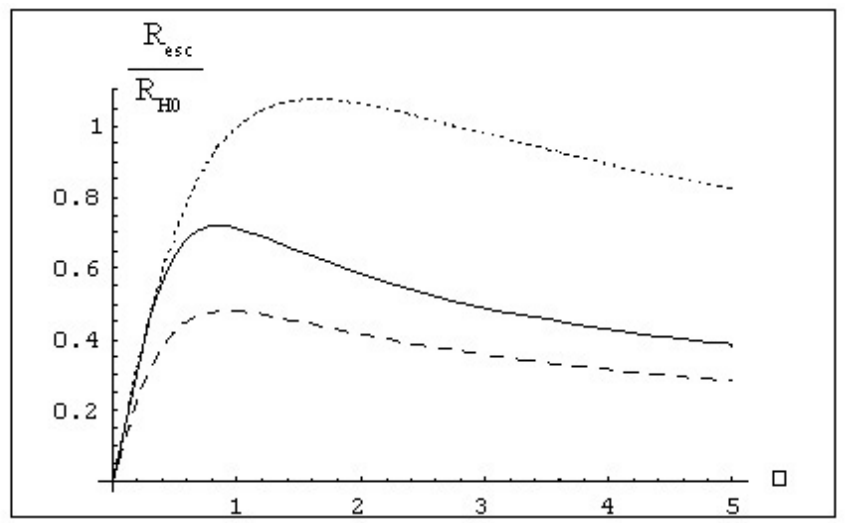

Figure 2: The comoving radius of escape in units of the comoving Hubble radius from Eq. (9). The solid line is the first, dashed line second, and the dotted line third of Eq. (9). The averaging process does make drastic difference; the third one that uses the rms speed grows to the greatest height. Although the curves shown are for flat universe, closed and open universe also show a similar trend of rise to a maximum till about equality, then slow decrease.

where, in the last equation, $\mathrm{P}$ stands for pressure and $\mathrm{v}^{2}$ has been replaced by $3 \mathrm{P} / \mathrm{\rho}$. The plot of $\mathrm{R}_{\text {esc }}$ averaged in the three ways of Eq. (9) are displayed in Fig.-2 for a flat universe, but the general trend for closed and open is also the same: $R_{\text {esc }}$ increases to a maximum value, and then decreases slowly with the expansion of the universe. But there are drastic differences in the values of the last average relative to the first two; e.g. for $\Omega_{\gamma \text { eq }}=\Omega_{v \text { eq }}=0.5$ that is shown, we find that the maximum of $\left(\frac{R_{R_{n}}}{\xi R_{H_{e}}}\right)_{\text {max }}=0.757$ occurs at $\xi=0.847$, that of $\left(\frac{R_{n+}}{s R_{H_{H}}}\right)_{2 \max }=0.477$ occurs at $\xi=0.922$, and $\left(\frac{R_{n}}{R_{i}}\right)_{3 \min }=1.074$ at $\xi$ $=1.632$. These different values indicate a spectrum of possible length scales. We may combine Eqs. (6) and (7) to write

$\frac{H_{H}}{R_{\alpha c}}=\sqrt{\frac{f_{s}(\xi)}{f_{\nu}(\xi)}}$

This relation displayed in Fig.-3, shows that the horizon crossing at $\mathrm{R}_{\mathrm{H}} / \mathrm{R}_{\text {esc }}=1$ occurs at different times for different averages. $R_{J}$, proportional to $R_{\text {esc }}$, will also show similar behavior.

The smallest of all these length scales, $R_{\text {esc }}$, enters the horizon the earliest, while the largest, $R_{\mathrm{J}}$, enters later. It appears that the time lag between $R_{\mathrm{J}}$ entering the horizon at the onset of Jeans instabilities, the virialization of the cosmic neutrino inside a smaller $R_{\mathrm{V}}$ that entered the horizon earlier, and even the Keplerization of the gas in an even smaller length $R_{\mathrm{K}}$ cannot be great.

We can also calculate the neutrino mass within $\mathrm{R}_{\text {esc }}$, viz. $M_{e x}=\frac{4 \pi}{3} p_{v} R_{e s c}^{3}$ that simplifies to

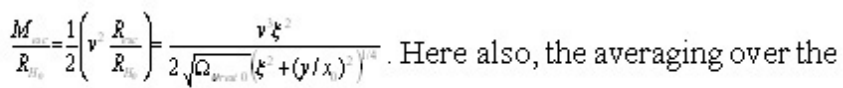
momentum of $\mathrm{M}_{\text {esc }}$ can be done in many different ways. Below, we give three which we think are useful: 


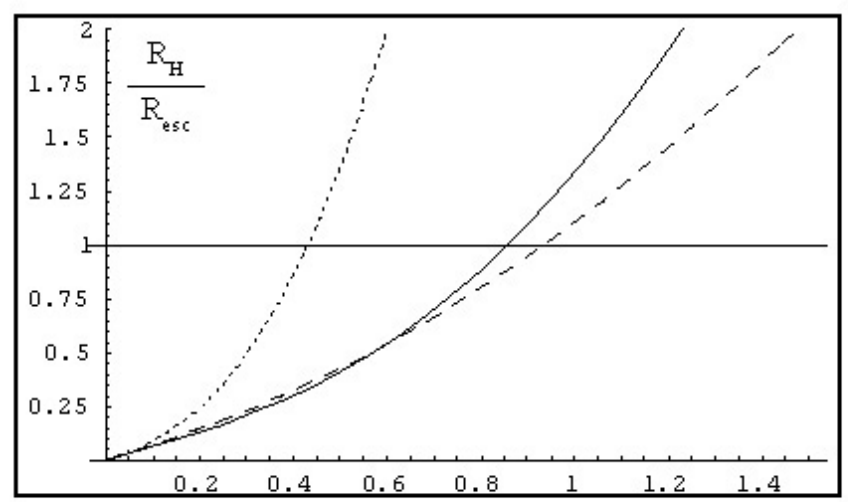

Fig.-3: The ratio of $\mathrm{R}_{\mathrm{H}} / \mathrm{R}_{\text {esc }}$ for the three averages against $\xi$. The third average enters the horizon the latest.

$$
\begin{aligned}
& \left\langle\frac{M_{o x}}{R_{H_{H}}}\right\rangle=\frac{1}{2 \sqrt{\Omega_{\nu N x}}}\left\langle\frac{\left(y / x_{0}\right)^{2} \xi^{2}}{\left(\xi^{2}+\left(y / x_{0}\right)^{2}\right)^{4}}\right\rangle \\
& \left\langle\frac{M_{o n}}{R_{H_{0}}}\right\rangle=\frac{1}{2}\left\langle v^{2}\right)\left(\frac{R_{\text {on }}}{R_{H_{0}}}\right\rangle=\frac{1}{2 \sqrt{\Omega_{\nu n a t 0}}}\left\langle\frac{\left(y / x_{0}\right)}{\xi^{2}+\left(y / x_{0}\right)^{2}}\right)\left(\frac{\left(y / x_{0}\right) \xi^{2}}{\left(\xi^{2}+\left(y / x_{0}\right)^{2}\right)^{4}}\right\rangle
\end{aligned}
$$

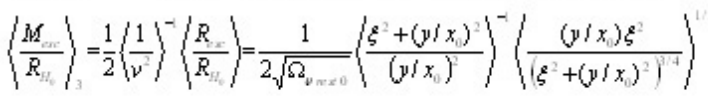

A plot of $\left(\mathrm{M}_{\text {esc }} / \mathrm{R}_{\mathrm{H} 0}\right)$ is shown in Fig. - 4 for three different averages. Although the nature of the curves are same, the peaks are seen to occur at different positions.

\section{FREE STREAMING}

There are two other scales that are relevant in this context. The first is the particle horizon $\mathrm{R}_{\mathrm{p}}$ occurring at the distance to which light would travel since the big bang. The comoving particle horizon can be written as

$$
\frac{1}{\xi} \frac{R_{p}}{R_{H_{u}}}=\int_{0}^{k} \frac{d \xi}{\sqrt{f_{u}\left(\xi^{j}\right)}}
$$

No massive particle can cover a distance larger than this. The other is the free streaming length $R_{F}$, the distance a particle travels since the big bang; it is given by an expression

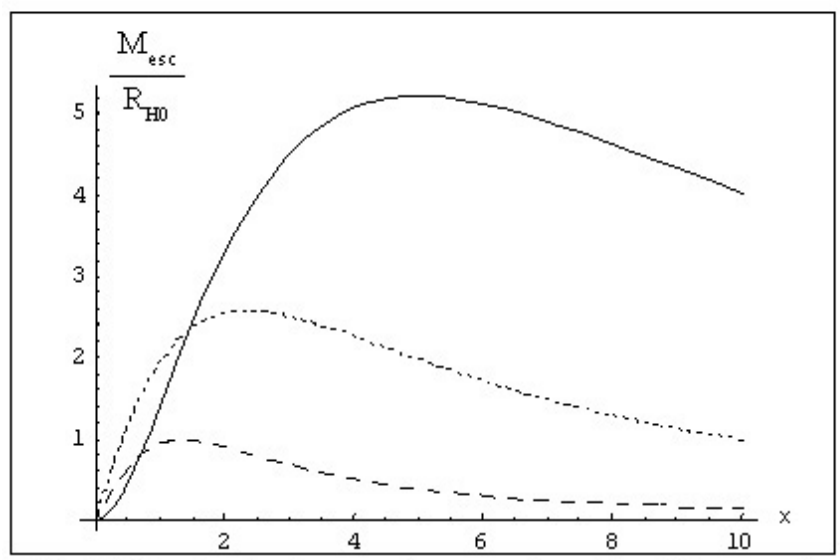

Fig.-4: The structure's mass for escape in units of the comoving Hubble radius from Eq. (11). The solid line is the first, dashed line second, and the dotted line third of Eq. (11). For all the curves the trends are the same, rise to a maximum, then slow decrease. But the position of the peak has significantly shifted. similar to [12], but including the particle speed $\mathrm{v}$ inside the integral

$$
\begin{aligned}
& \frac{1}{\xi} \frac{R_{F}}{R_{H_{0}}}=\int_{0}^{\xi} \frac{v d \xi^{\prime}}{\sqrt{f_{u}\left(\xi^{\prime}\right)}}
\end{aligned}
$$

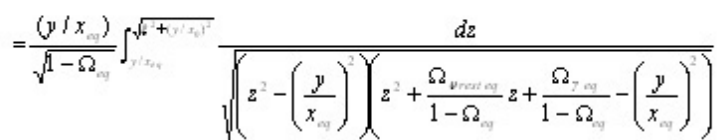

Any scale that is smaller than $R_{F}$ will be wiped out by free streaming. The integral Eq. (14) in our case, for different values of $\Omega_{y e q}$, can be written as Jacobian elliptic functions $\left[{ }^{10}\right],\left[{ }^{11}\right]$

$$
\left(\frac{1}{\xi} \frac{R_{F}}{R_{H_{k}}}\right)_{\text {flut }}=C F(\phi / k)
$$

Considering the flat case where $\Omega_{\text {yeq }}=0.5$, the solution is given by $C^{2}=\frac{8\left(y i x_{0 v}\right)^{2}}{1+\frac{3 \zeta(3) y}{4 \zeta(4)}\left(\frac{T_{v}}{T_{7}}\right)^{4}}$, $\sin ^{2} \phi=\frac{\sqrt{x^{2}+y^{2}}-y}{\sqrt{x^{2}+y^{2}}+y}$, and $k^{2}=\frac{\sqrt{x_{x}{ }^{2}+y^{2}}-x_{\infty}}{\sqrt{x^{2}+y^{2}}+x_{\infty}}$. Aplot of $\left(\mathrm{R}_{\mathrm{F}} / \xi_{\mathrm{R}} \mathrm{H}_{\mathrm{eq}}\right)_{\mathrm{flat}}$ is shown in Fig. 5 for three different mean values of $y$. This free streaming length is seen to saturate to the values of 4.193 , 3.899 and 2.555 as $\mathrm{x} \rightarrow \infty$, when evaluated with $\mathrm{y}_{\mathrm{ms}}, \mathrm{y}_{\mathrm{mean}}$ and $\mathrm{y}_{\text {rims }}$ respectively.

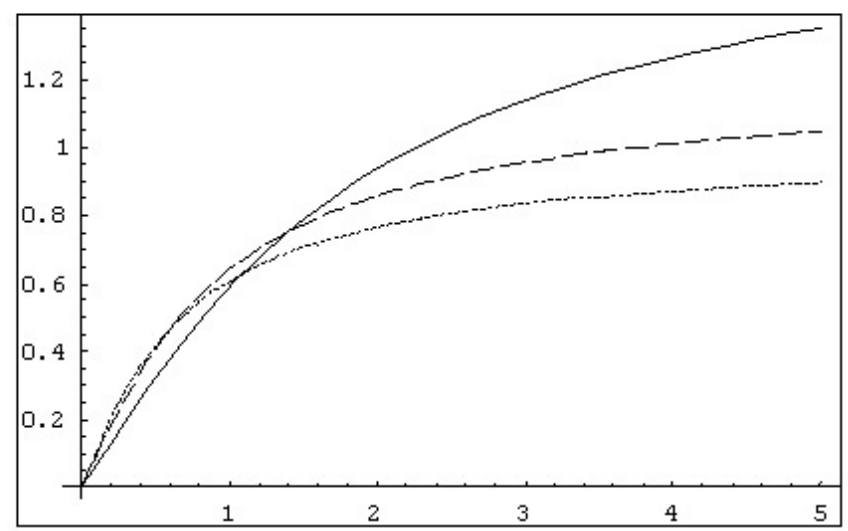

Fig.-5: A plot of $\left(\mathrm{R}_{\mathrm{F}} / \xi \mathrm{R}_{\mathrm{H}} \text { eq }\right)_{\text {flat }}$ against $\xi$ for three different mean values of $y$. The solid line is for mean, dashed line from $v_{m s}$ and the dotted line from $v_{\text {inms }}$

\section{DISCUSSION AND CONCLUSION}

The effect of massive neutrino on large structures has been investigated. The scale of neutrino structures appear to depend on the average speeds of the particles. Particularly, in Jeans analysis, the fundamental quantity is the Jeans wave vector that depends on the reciprocal of the average velocity. So the actual size of those neutrino structures appear to be very sensitive to the averaging procedure used for the velocity. So there exists a distribution of these scales with quite different values. 
In the case of length, the peak values of different averages of $\left(\frac{R_{x}}{\xi R_{H_{e}}}\right)$ are $0.477,0.757$ and 1.074 occurring at $\xi=0.92,0.847$ and 1.63 respectively. Similarly, peak values of different averages of free-streaming lengths for flat universe are 2.555 , 3.899 and 4.19 at large $\xi$. The maxima of different average masses of the neutrino structures are $0.98,2.57$ and 5.20 which occur at the instants of $\xi=1.34,2.35$ and 5.7 respectively.

\section{REFERENCES}

[1] Steigman, G., Schramm, D. N. and Gunn, J.E. 1977. Phys. Lett. 66 B. p. 202.

[2] Winter, K. 2000. Neutrino Physics. Cambridge University Press. p. 197.

[3] Yao, W.M. et al. 2006. J. Phys. G33.
[4] Cowsik, R. and McClelland, J. 1972. Phys. Rev. Letter. 29:669.

[5] Treamine, S. and Gunn J.E. 1979. Phys. Rev. Letter. 42:407.

[6] Bond, J.R., Efstathiou, G and Silk, J. 1980. Phys. Rev. Letter. 45: 1980 .

[7] Spergel, D.N., et al. arxiv: astro-ph/0603449

[8] Zeldowich, Y.B. Eiviasto, J. and Shandarin, S. F. 1982. Nature 300:407.

[9] Abramowitz, M. and Stegun, I. A. Handbook of Mathematical Functions, Dover, New York.

[10] Byrd, P.F. and Friedman, M.D. 1971. Hand Book of Elliptic Integrals for Engineers and scientists. Springer, Berlin.

[11] Gradshteyn, I. S. and Ryzhik, I. M. 1993. Tables of Integrals, Series and Products. Sth ed. Academic, Boston. 